\title{
Assessing the relationship self-medication with chronic condition self-medication: analytic- descriptive study.
}

Hesam Alidn Varpaei ( $\square$ akademik.hv@gmail.com )

Islamic Azad University https://orcid.org/0000-0002-6923-2040

Pariya Onsori

Islamic Azad University

Faeze Esmaeili

Islamic Azad University

Saba Abachi

Islamic Azad University

Pedram Nouroozi

Islamic Azad University

Amir Mahdi Farahani

Islamic Azad University

Mohammad Mahdi Miremami

Islamic Azad University

Ali Kazami

Islamic Azad University

\section{Research Article}

Keywords: Chronic condition, self-medication, health promotion, adherences, cancer

Posted Date: November 30th, 2020

DOI: https://doi.org/10.21203/rs.3.rs-110942/v2

License: (c) (i) This work is licensed under a Creative Commons Attribution 4.0 International License.

Read Full License 


\section{Abstract}

Background: Chronic disease is a stable condition of human health that refers to the disease or its effects or the effects that occur over time. Lack of awareness in chronic patients can be challenging, as it provides the basis for self-medication. This study was performed to investigate the prevalence of selfmedication in people with chronic disease and the factors affecting it.

Materials and Methods: A cross-sectional study was conducted in Tehran from January to March 2020 and targeted people living in Tehran.

Results: The prevalence of self-medication in healthy individuals was $64 \%$ and in chronic patients was $90 \%$. The most important reason for self-medication in patients was having an old prescription and easy availability in healthy individuals. Analgesics and cold medications were the most commonly used. Headache, muscle aches, and runny nose were the most common symptoms / illnesses of selfmedication. Self-medication was significantly associated with chronic illness, duration of disease, number of chronic disease medications, and study of medication instructions $(P<0.05)$.

Conclusion: The prevalence of self-medication among people with chronic diseases is significantly higher due to having a previous prescription or for economic reasons such as the high price of doctor visits and this is a health problem that needs to be addressed. Income, smoking, duration of chronic illness, number of medications taken by the patient and reading medication instructions (drug catalog or brochure) are some of the factors affecting self-medication. One of the simple steps for health promotion in chronic conditions is to educate chronic patients properly that might mitigate the complications of selfmedication and prevent dire consequences of inappropriate medication use.

\section{Background}

The emergence of chronic diseases as the most important challenge to global health is undeniable [1]. Chronic disease is a stable state of human health that refers to the disease or its effects or effects that occur over time. In fact, it is a lifelong disease to death [2].

The World Health Organization states that chronic diseases are not transmitted from person to person. These types of diseases are long in nature and generally progress slowly. The four main types are cardiovascular disease (such as heart attack and stroke), cancer, chronic respiratory disease (such as chronic obstructive pulmonary disease and asthma), and diabetes. Other types include mental illness, Alzheimer's, cerebral palsy, HIV / AIDS, obesity, and thyroid disorders [3] [4]. In addition, the World Health Organization predicts that total annual deaths from the chronic disease will reach 52 million by 2030 [5]. Chronic disease can occur at any age, although in some stages of life such as aging are associated with greater risks. Aging is one of the most important stages of life and in the near future, a large percentage of the Iranian population will be elderly. This period of life is often associated with an increased risk of chronic diseases [6]. 
Studies show that patients with low health literacy and chronic diseases such as diabetes, asthma, or hypertension are less aware of the disease and its treatment, and also have less proper self-management skills than knowledgeable patients [7]. Lack of awareness in patients with a variety of chronic diseases can be challenging, as it provides the basis for self-medication.

The World Health Organization defines self-medication as the selection and use of over-the-counter medications without the supervision of a physician or dentist. In general, self-medication is defined as "the use of medications to treat self-diagnosed disorders or symptoms or the intermittent or continuous use of a medication prescribed for a disease or chronic or recurrent symptoms" [8] [9].

There are several reasons for self-medication, the results of a review study, minor illness, health care costs, not having enough time to see a doctor, previous experience with medication, and long waiting times to see a specialist have been the most reported causes of self-medication [10]. Another study states that most over-the-counter medications were analgesics and antibiotics, respectively. Headache, allergies, and fever were the most common symptoms of self-medication. Female gender, older age, maternal education, and family habits were among the causes of self-medication. The main sources of information about drugs have been pharmacists, parents, and friends [11]. Another study confirms that age, sex, and level of education, and income were the main determinants of self-medication, and social, cultural, economic, and regulatory factors were the most common reasons for self-medication. The main sources of drug information included relatives/friends and previous successful experience [12]. The results of a meta-analysis specifically state that the main determinants of self-medication with antibiotics were education, age, sex, past successful use, disease severity, and income [13].

Patients with chronic illnesses should take medication to control or prevent disease progression. Use continued several medications can provide a psychological basis for self-medication so that patients are more likely to take one or more medications as self-medication. However, improper use of drugs and side effects of drugs can lead to interaction with chronic disease control drugs and even lead to the progression of the disease imperceptibly. Therefore, the aim of this study was to investigate the relationship between self-medication and chronic disease.

\section{Method And Material}

The present study was a cross-sectional descriptive-analytical study that was conducted in the winter of 1398 (January to March 2020) on community members (including students, ordinary people, businesses, passers-by referring patients to clinics and hospitalized patients) in Tehran.

After designing the questionnaire, individuals were included in the study with the condition of consent to participate in the project. All questionnaires were anonymous. Participants were assured of confidentiality. The questionnaire consisted of 30 questions in the form of three axes of personal information, physical activity pattern, smoking or alcohol consumption, and self-medication habits in the last 6 months. Each questionnaire was numbered and coded information was extracted. 
In this study, the criterion for considering a chronic disease is a condition or disease that has no definitive cure and a person must have health care (such as taking medicine, receiving blood products) for the rest of his life to control and control the disease.

SPSS 23 software was used for statistical analysis. The significance level of the data was considered $5 \%$. Pearson correlation coefficient test was used for the relationship between quantitative variables. Also, a one-way analysis of variance and Tukey post hoc test was used.

\section{Results}

The demographic information of the participants in this study is given in Table 1 . The mean age of participants was $62 \pm 39,495$ participants were single and 305 were married. 218 participants had chronic disease/condition. The rate of self-medication was 64\% (372 people) in people without chronic disease and $90 \%$ (196 people) in people with chronic disease.

\section{Table 1 - Demographic information of the participants}

\begin{tabular}{|c|c|}
\hline Sex & Frequency (percentage) \\
\hline Male & $360(45 \%)$ \\
\hline Female & $440(55 \%)$ \\
\hline \multicolumn{2}{|c|}{ Education } \\
\hline Diploma or less & $438(54.75 \%)$ \\
\hline College education & $362(45.25 \%)$ \\
\hline \multicolumn{2}{|c|}{ Chronic condition/disease } \\
\hline Yes & $218(27.25 \%)$ \\
\hline No & $582(72.75 \%)$ \\
\hline \multicolumn{2}{|c|}{ Duration of Chronic condition/disease } \\
\hline 1 to 2 years & $73(33.5 \%)$ \\
\hline 2 to 5 years & $79(36 \%)$ \\
\hline 5 to 10 years & $32(14.5 \%)$ \\
\hline More than 10 years & $34(16 \%)$ \\
\hline \multicolumn{2}{|c|}{ Number of medications taken for Chronic condition/disease } \\
\hline 1 to 2 pieces & $109(50 \%)$ \\
\hline 2 to 5 pieces & $72(33 \%)$ \\
\hline 5 to 10 pieces & $25(11.5 \%)$ \\
\hline More than 10 pieces & $12(5.5 \%)$ \\
\hline
\end{tabular}




\begin{tabular}{rc}
\hline \multicolumn{3}{c}{ Table 2 - Self-medication in different groups } \\
\hline \multicolumn{3}{r}{ People with chronic condition/diseases } & Frequency (percentage) \\
\hline Female & $100(51 \%)$ \\
\hline Male & $96(49 \%)$ \\
\hline People without chronic condition/diseases \\
\hline Female \\
\hline Male \\
\hline Total & $154(518 \%)$ \\
\hline
\end{tabular}

The prevalence of chronic diseases is 83 patients with diabetes, 74 patients with heart disease and hypertension, 17 patients with respiratory diseases (such as asthma, chronic obstructive pulmonary disease), 16 patients with gastrointestinal diseases (such as gastroesophageal reflux disease), and 6 patients had other diseases (such as cancer, skin diseases, etc.).

Of the study population, 608 did not smoke at all and 192 smokers. Of these, 122 smokers, 65 used hookah and 5 used marijuana.

$88(11 \%)$ of the study population (including 24 with chronic disease and 64 non-chronic diseases) had a history of drug allergy. The most important allergens were: penicillins (40\%), Aspirin (25\%), corticosteroids $(10 \%)$, anticonvulsants (5\%), and nonsteroidal analgesics (20\%).

There were several criteria for choosing a drug by healthy people (without chronic disease), the results showed: easy access 141 people (38\%), pharmaceutical company 108 people (29\%), price 93 people (25\%) and pharmaceutical brand 30 people (8\%) were the most frequent reasons.

However, people with chronic disease stated that medication prices (58\%), pharmaceutical companies (32\%), and easy access or availability (10\%) were the most important criteria for drug selection, respectively.

A wide range of medications has been used by healthy and sick people for self-medication. Among the analgesics, acetaminophen, ibuprofen (Advil), and aspirin, respectively, gastrointestinal drugs including ranitidine, omeprazole, and ondansetron (demitron), cold medicines including cold gel and adult cold were the most used drugs. The most commonly used antibiotics were amoxicillin (38\%), cephalexin $(23 \%)$, azithromycin $(21 \%)$, and cefixime $(15 \%)$, respectively. Other antibiotics accounted for $3 \%$ of all selfmedication cases. All participants used more than one medication for self-medication. 
Table 3 - Used medication for self-medication

\begin{tabular}{ccc}
\hline & Frequency & Relative frequency percentage \\
\hline Analgesics & 482 & $84.8 \%$ \\
\hline Cold medicines & 412 & $72.5 \%$ \\
\hline Gastrointestinal drugs ** & 363 & $63.9 \%$ \\
\hline Multivitamins and minerals & 312 & $54.9 \%$ \\
\hline Antibiotics & 296 & $52.1 \%$ \\
\hline Antihistamines & 265 & $46.6 \%$ \\
\hline Antitussives and expectorants & 240 & $42.2 \%$ \\
\hline Anti-inflammatory drugs & 174 & $30.6 \%$ \\
\hline Sedations & 96 & $16.9 \%$ \\
\hline Skin medications & 56 & $9.8 \%$ \\
\hline Ophthalmic drugs & 38 & $6.6 \%$ \\
\hline Contraceptive drugs & 32 & $5.6 \%$ \\
\hline *For example: Adult Cold or Cold Gel & \\
**Includes anti-acid drugs, anti-emetic and anti-diarrhea drugs.
\end{tabular}

The most commonly used form of medication (Figure 2) in all participants was tablets and capsules, followed by ampoules. Although most forms of drugs available in pharmacies are in these two forms, injectable drugs often require special care and must be injected in medical centers under the supervision of a physician. It should also be noted that not all used medications (in any form) included over-thecounter medications.

The illnesses for which individuals attempted self-medication are listed in Table 4. It is worth noting that all participants referred to more than 1 case. 


\begin{tabular}{rcc}
\hline $\begin{array}{c}\text { Table 4 - Frequency of self-medicated symptoms } \\
\text { or diseases }\end{array}$ & Frequency & $\begin{array}{c}\text { Relative frequency } \\
\text { percentage }\end{array}$ \\
\hline Headaches and migraines & 325 & $57.2 \%$ \\
\hline Muscular pain & 295 & $51.9 \%$ \\
\hline Runny nose (rhinorrhea) & 276 & $48.5 \%$ \\
\hline Cough & 263 & $46.3 \%$ \\
\hline Nausea and vomiting & 178 & $33.3 \%$ \\
\hline Diarrhea & 104 & $18.3 \%$ \\
\hline Toothache & 88 & $17.2 \%$ \\
\hline Fever & 80 & $15.3 \%$ \\
\hline Heart burn (gastroesophageal relux) & $14 \%$ \\
\hline Mouth sores & 50 & $8.8 \%$ \\
\hline Blood pressure & 25 & $4.5 \%$ \\
\hline 21 & $3.4 \%$ \\
\hline Whortness of breath and asthma & $3.6 \%$ \\
\hline Wounds and injuries & 20 & $3.5 \%$ \\
\hline Dair loss and dandruff & 19 & $3.3 \%$ \\
\hline Diabetes & 15 & $2.6 \%$ \\
\hline Urinary problems & 8 & $1.4 \%$ \\
\hline Contraception & 4 & $0.7 \%$ \\
\hline Skin disease & 4 & $0.7 \%$ \\
\hline
\end{tabular}

The most common self-medicated diseases (in healthy and sick people) are headache and migraine, muscle pain (myalgia), and cough, respectively. It is clear that according to the results, the most common illness was cold and symptomatic treatment. However, due to the sale of over-the-counter drugs in most pharmacies in Tehran, self-medication is also seen in chronic diseases such as hypertension, asthma, and diabetes.

$297(80 \%)$ of healthy people who self-medicated read the medication instructions and information (written on the medication, catalog, or medicine box) before taking the medication. 75 (20\%) did not read the medication. Among chronic patients who self-medicated, only $72(36 \%)$ stated that they read medication instructions and information before use, and 124 (64\%) did not check medication information before taking medication. In healthy individuals, 140 people (37.5\%) experienced drug side effects. The most common complications were diarrhea, nausea, vomiting, and headache. In patients, this amount was reported in only 10 patients $(5.1 \%)$.

Based on Pearson correlation coefficient analysis, there was a significant relationship between chronic disease and self-medication $(r=0.22$ and $P$-Value $=0.00)$, number of medications and chronic disease $(r$ $=0.23$ and $\mathrm{P}$-Value $=0.00)$ and duration of chronic disease and self-medication $(r=0.48$ and P-Value $=$ 0.00 ). Smoking behavior (like cigarette or tabaco use) was also significantly correlated with selfmedication $(r=0.21$ and $P$-Value $=0.00)$. However, there was not a significant correlation between gender and self-medication ( $p$-Value $=0.49, r=0.67$ ), exercise and self-medication ( $p$-Value $=0.18, r=0.79$ ), alcohol consumption and self-medication ( $p$-Value $=0.77, r=0.39$ ). Although the study of information and prescription was significantly correlated with self-medication in healthy individuals ( $p$-Value $=0.006$, 
$r=0.18$ ), in the group of patients the reading the medication information and prescription was not significantly associated with self-medication ( $p$-Value $=0.48, r=0.46)$.

One-way analysis of variance was used to compare the mean of the data and self-medication. Tukey post hoc test was used to follow the differences between the groups. Self-medication was significantly higher $(P$-value $=0.00)$ in people with chronic diseases $(75 \pm 12)$ than in people without chronic diseases $(27 \pm 14)$. The mean self-medication of people who took 1 to 2 drugs for chronic disease (16 \pm 5$)$ was higher than those who took 5 to 10 drugs for chronic disease $(14 \pm 1)$ and those who took 2 to 5 drugs for chronic disease $(9 \pm 1)$ and this difference was statistically significant $(P$-value $=0.00)$. However, people who did not take any medication for chronic disease were on average less than other people who took some medications $(-3 \pm 1)$ and this difference was significant (P-value $=0.005)$. People who did not read the medication information and prescription $(10 \pm 1.25)$ on average self-medicated more than other people $(1 \pm 0.2)(P$-value $=0.00)$

\section{Discussion}

Self-medication is defined as taking medicine without a doctor's prescription, and this is a public health problem worldwide, especially in countries with limited resources. Although self-medication can reduce waiting time and save costs, it might carry some potential risks [14].

One of the main challenges in the discussion of self-medication is to address it in people with chronic diseases. Chronic illness is a major problem in the field of health care and social services. For this reason, people with chronic diseases need more health consideration because of the chronic condition features that increase their vulnerability.

It seems that in addition to the nature of chronic diseases, some behavioral features as well as lifestyle are effective in the incidence of self-medication in people with the disease. Smoking, inadequate physical activity, and lack of attention to nutrition increase the risk of various chronic diseases [15]. For this reason, one of the main causes of public health monitoring for many years, the system of monitoring behavioral risk factors affected by the occurrence of chronic diseases [16].

For example, since chronic obstructive pulmonary disease (COPD) is one of the most debilitating diseases and the third leading cause of death worldwide, the findings suggest that some self-care behaviors such as drug adherence, physical activity, and Cigarettes withdrawal as well as the level of knowledge of individuals have a great impact on the rate of recovery [17]. Therefore, it seems that people's habits are also effective in self-care and self-medication. For this reason, according to studies conducted on people in this study, most people had not any smoking habit, which is considered optimistic.

In addition, demographic characteristics of people such as age, sex, level of education, and income can also affect the incidence of self-medication in patients. In a study, it was found that people with an average income level (between 2-5 million Tomans) were the most people who self-medicated [18]. 
Although past experiences do not indicate a significant relationship between self-medication and income level, attitudes to this issue seem to differ today.

Age is a major predictor of many chronic diseases, with the highest incidence of disease, hospitalization, health costs, and mortality worldwide. In such a way that their occurrence at a young age and old age is challenging. These include Alzheimer's and other diseases of the nervous system, cardiovascular disease (hypertension), and most cancers. Aging, in addition to the problems it causes, increases the prevalence of chronic diseases and even the occurrence of several diseases simultaneously in a person [19].

Failure to follow medication is an important element of uncontrolled blood pressure [20]. Few studies have examined the association between drug literacy and adherence in patients with hypertension [21]. It seems that because drug literacy and drug adherence are not significantly related, the risk of selfmedication in these people is much higher. On the other hand, self-medication in these conditions poses more risks to a person's health due to the possibility of interactions with blood pressure medications. As a result, adherence to the medication regimen is considered important in the event of several diseases occurring simultaneously (chronic and acute). Therefore, non-compliance with prescribed drugs is associated with adverse health consequences in people with chronic diseases [22]. Among the factors that lead to poor adherence with prescriptions include the simultaneous use of several drugs (polypharmaceuticals) that include prescription and over-the-counter drugs, demographic characteristics, and health care systems [23]. Non-adherence to the medication regimen in children with chronic disease who use them for a long time is a serious problem and assessing adherence is an important step in providing a solution to this problem [24]. Due to the nature of the disease and its effects on family members, or even fatigue caused by frequent visits to the doctor, it is possible for parents to resort to arbitrary medication to quickly eliminate minor symptoms in the child.

Also, the elderly with chronic diseases, due to the aging process that causes changes in their mobility, and on the other hand, due to being prone to osteoporosis and cognitive changes due to dementia, create conditions for them to prevent or modify symptoms as recommended by others to take a variety of medications, minerals, and supplements. However, depending on the type of chronic disease and the medications used, they might cause side effects and cause them health consequences and even lifethreatening problems. For this reason, it is recommended that patients read the medications brochure before taking any medication as much as possible to be aware of the potential risks, side effects, and how to take the medication. Since in this study, a small number of patients have read the instructions before taking the medications, it would be problematic and might have dire health consequences. For this reason, one of the important tasks of health care providers is to inform people about the disease not to use drugs arbitrarily, and then encourage them to read medication prescriptions.

Self-efficacy is needed to manage chronic diseases. Since one of the cases of turning to self-medication can be insufficient information and misunderstanding of the deterioration of the current situation, therefore in this situation, the person needs to be taken care of by an informed person. Accordingly, in these cases, physicians, in collaboration with patients and their caregivers, assess the patient's current 
ability and identify barriers to self-care and try to provide information to their caregivers based on the patient's skills and abilities [25].

Based on the available evidence, effective self-care can improve health and improve quality of life and reduce the need for health care [26]. However, resorting to self-medication under the guise of self-care can have the opposite effect. According to this study, one of the most common causes of self-medication in patients is having an old doctor's prescription and saving time. It seems that due to the nature of the chronic disease and its duration and the number of medications taken, patients prefer to resort to selfmedication instead of consulting with a doctor for minor problems such as cold symptoms, types of pain, digestive problems, etc. Other reasons for the high cost of a doctor's visit are lack of trust in doctors and the lack of access to a doctor or clinic. Due to these factors, a person's income can also have a positive effect on the incidence of self-medication. Especially if the person does not have a support system. This is confirmed by the fact that most people with chronic diseases have stated the price of the medications.

Due to the fact that the most common form of medicine is pills and capsules, as a result of these forms, they are more self-medicated because they do not need special skills to use and also do not need special care after consumption. For this reason, they are relatively easier to use than other forms of medication, even by people with a chronic disease who do not know enough about drugs or have a low level of education.

\section{Conclusion}

Self-medication was commonly used in people with chronic diseases, significantly more than people without chronic disease. The most common reason among them was having a previous prescription or socioeconomic reasons such as the high cost of doctor consultations. Self-medication is mostly used to relieve symptoms such as headaches or cold symptoms. Level of income, smoking habit, duration of chronic disease, number of medications taken by the patient, and reading medication instructions (drug catalog or brochure) were some of the factors affecting self-medication. Health care professionals, such as doctors and nurses, must play their part by providing guidance and counseling to their patients on the proper use of medications. Educational programs aimed at people with chronic diseases and their families are also needed to inform people about the dire consequences and adverse effects of selfmedication in chronic diseases. Another important intervention to reduce the major problem of selfmedication should be legal changes that prohibit the overselling of medication that requires physician prescription. Finally, it seems that increasing the support of health insurance services and maximum coverage of physician consultation costs can play an effective role in the prevention of self-treatment in chronic disease patients. One of the simple steps for health promotion in chronic conditions is to educate chronic patients properly that might mitigate the complications of self-medication and prevent dire consequences of inappropriate medication use.

\section{Declarations}




\section{Conflicts of Interest}

The authors declare no conflicts of interest.

Ethics: This study was approved by ethic committee of Islamic Azad University Tehran medical sciences.

Ethic code: lautmu 1398.124 .12

\section{References}

1 - World Health Organization. "WHO| United Nations high-level meeting on noncommunicable disease prevention and control." (2015).

2 - Bernell, Stephanie, and Steven W. Howard. "Use your words carefully: what is a chronic disease?." Frontiers in public health 4 (2016): 159.

3 - Hunter, David J., and K. Srinath Reddy. "Noncommunicable diseases." New England Journal of Medicine 369.14 (2013): 1336-1343

4 - World Health Organization. World health statistics 2016: monitoring health for the SDGs sustainable development goals. World Health Organization, 2016.

5 - World Health Organization. Investing to overcome the global impact of neglected tropical diseases: third WHO report on neglected tropical diseases 2015. Vol. 3. World Health Organization, 2015

6 - Maghsoudi, Ahmad. "The study of prevalence of chronic diseases and its association with quality of life in the elderly of Ewaz (South of Fars province), 2014." Navid No 18.61 (2016): 35-42

7 - Gazmararian, Julie A., et al. "Health literacy and knowledge of chronic disease." Patient education and counseling 51.3 (2003): 267-275.

8 - Awad, Abdelmoneim, et al. "Self-medication with antibiotics and antimalarials in the community of Khartoum State, Sudan." J Pharm Pharm Sci 8.2 (2005): 326-331.

9 - Bennadi, Darshana. "Self-medication: A current challenge." Journal of basic and clinical pharmacy 5.1 (2013): 19.

10 - Shaghaghi, Abdolreza, Marzieh Asadi, and Hamid Allahverdipour. "Predictors of self-medication behavior: a systematic review." Iranian journal of public health 43.2 (2014): 136.)

11 - Shehnaz, Syed llyas, Anoop Kumar Agarwal, and Nelofer Khan. "A systematic review of selfmedication practices among adolescents." Journal of adolescent health 55.4 (2014): 467-483.

12 - Alhomoud, Faten, et al. "Self-medication and self-prescription with antibiotics in the Middle East-do they really happen? A systematic review of the prevalence, possible reasons, and outcomes." International 
Journal of Infectious Diseases 57 (2017): 3-12.

13 - Ocan, Moses, et al. "Household antimicrobial self-medication: a systematic review and meta-analysis of the burden, risk factors and outcomes in developing countries." BMC public health 15.1 (2015): 742.

14 - Zeid, W., Hamed, M., Mansour, N. and Diab, R., 2020. Prevalence and associated risk factors of selfmedication among patients attending El-Mahsama family practice center, Ismailia, Egypt. Bulletin of the National Research Centre, 44(1), pp.1-5.

15 - Lankarani, Nargess Bagheri, et al. "Chronic diseases in a population-based study: Iranian Health Perception Survey (IHPS)." Payesh (Health Monitor) 10.3 (2011): 391-395.

16 - Birkhead, G.S., 2017. Successes and continued challenges of electronic health records for chronic disease surveillance.

17 - Hosseinzadeh, H. and Shnaigat, M., 2019. Effectiveness of chronic obstructive pulmonary disease self-management interventions in primary care settings: a systematic review. Australian Journal of Primary Health, 25(3), pp.195-204.

18 -Bilal, Muhammad, et al. "Self-medication with antibiotics among people dwelling in rural areas of Sindh." Journal of clinical and diagnostic research: JCDR 10.5 (2016): OC08.

19 - Tchkonia, T. and Kirkland, J.L., 2018. Aging, cell senescence, and chronic disease: emerging therapeutic strategies. Jama, 320(13), pp.1319-1320.

20 - Fang, J., Chang, T., Wang, G. and Loustalot, F., 2020. Association between cost-related medication nonadherence and hypertension management among US adults. American journal of hypertension.

21 - Shi S, Shen Z, Duan Y, Ding S, Zhong Z. Association Between Medication literacy and medication adherence among patients with hypertension. Frontiers in Pharmacology. 2019;10.

22 - Anoopkumar-Dukie, Shailendra, Amary Mey, Susan Hall, Nijole Bernaitis, Andrew K. Davey, and David Plummer. "Non-prescription medicines may contribute to non-adherence to prescription medicines in people living with chronic health conditions." International Journal of Clinical Practice 74, no. 6 (2020): e13489.

23 - Al-Hassany, L., Kloosterboer, S.M., Dierckx, B. and Koch, B.C., 2019. Assessing methods of measuring medication adherence in chronically ill children-a narrative review. Patient preference and adherence, 13, p.1175.

24 - Ibrahim, J.E., Anderson, L.J., MacPhail, A., Lovell, J.J., Davis, M.C. and Winbolt, M., 2017. Chronic disease self-management support for persons with dementia, in a clinical setting. Journal of multidisciplinary healthcare, 10, p.49. 
25 - Allegrante, J.P., Wells, M.T. and Peterson, J.C., 2019. Interventions to support behavioral selfmanagement of chronic diseases. Annual review of public health, 40, pp.127-146.

\section{Figures}

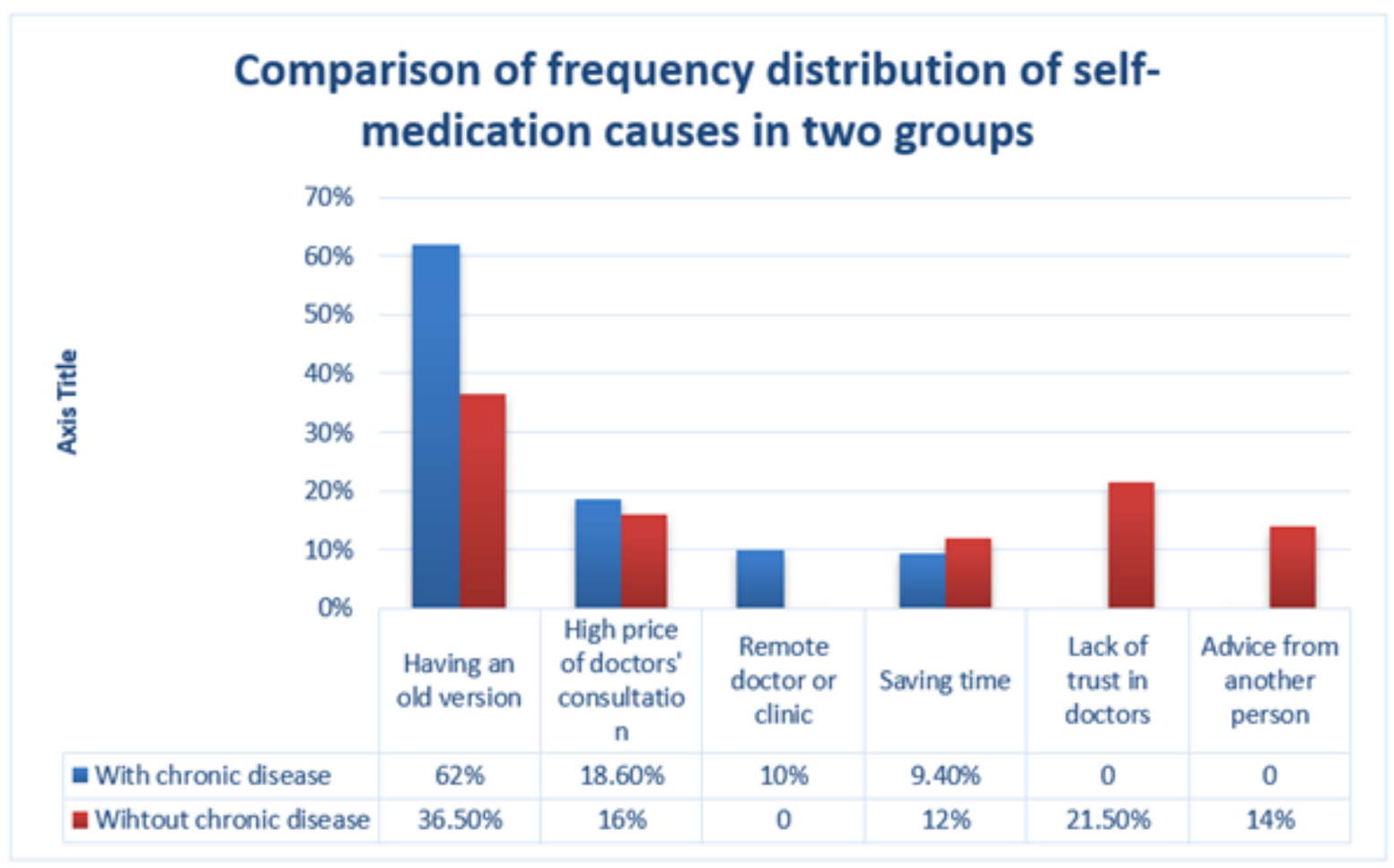

Figure 1

Comparison of frequency distribution of self medication causes in two groups 


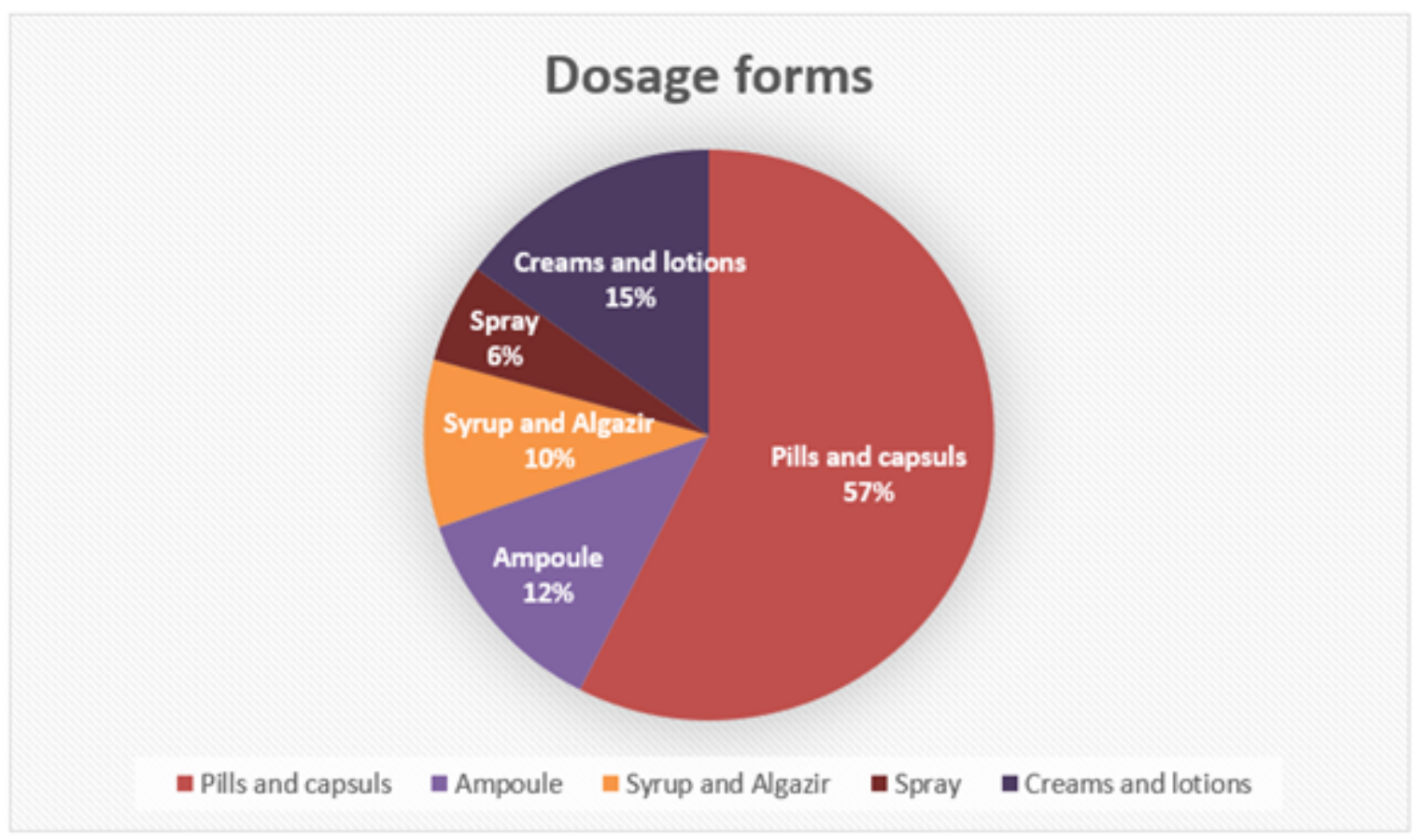

Figure 2

Dosage forms 